\title{
Torsional vibration of a shafting system under electrical disturbances
}

\author{
Ling Xiang, Shixi Yang and Chunbiao Gan* \\ Department of Mechanical Engineering, Zhejiang University, Hangzhou, Zhejiang, China
}

Received 24 May 2011

Accepted 14 November 2011

\begin{abstract}
Torsional vibration responses of a nonlinear shafting system are studied by a modified Riccati torsional transfer matrix combining with the Newmark- $\beta$ method. Firstly, the system is modeled as a chain consisting of an elastic spring with concentrated mass points, from which a multi-segment lumped mass model is established. Secondly, accumulated errors are eliminated from the eigenfrequencies and responses of the system's torsional vibration by this newly developed procedure. The incremental transfer matrix method, combining the modified Riccati torsional transfer matrix with Newmark- $\beta$ method, is further applied to solve the dynamical equations for the torsional vibration of the nonlinear shafting system. Lastly, the shafting system of a turbinegenerator is employed as an illustrating example, and simulation analysis has been performed on the transient responses of the shaft's torsional vibrations during typical power network disturbances, such as three-phase short circuit, two-phase short circuit and asynchronous juxtaposition. The results validate the present method and are instructive for the design of a turbo-generator shaft.
\end{abstract}

Keywords: Transfer matrix method, Newmark- $\beta$ method, turbo-generator shaft, electrical disturbance, torsional vibration

\section{Introduction}

Rotor dynamics plays an important role in many engineering fields, such as gas turbine, steam turbine, reciprocating and centrifugal compressors, the spindle of machine tools, and so on. Owing to the growing demands for high power of the rotor system, computations of critical speeds and dynamical response become essential for system design, identification, diagnosis, and control. Due to the turbine rotor failures at Southern California Edison's Mohave station in 1970 and 1971 [1], industry's attention was focused on the shaft's torsional vibration caused by the transmission operation in a turbine-generator. The shafting system of modern large turbine-generator units is a high speed coaxial solid of revolution. It is connected by elastic-coupling parts, and composed of the turbine rotors, generator and exciter. An electromechanical transient process caused by power system failure or changes of operating conditions may lead to the shaft's torsional vibration, which is very important for shafting system designers. Fundamental to the study of turbo-generator shaft torsional vibrations, as occurs in subsynchronous resonance and high speed reclosing, is the calculation of the characteristic frequencies and responses of the vibrations.

Currently, the finite element and transfer matrix approaches are two of the most prevalent methods for analyzing torsional vibration of shafting systems. The finite element method (FEM) formulates rotor systems by second-order differential equations directly utilized for control design and estimation, while the transfer matrix method (TMM) solves dynamical problems in the frequency domain [2]. The TMM utilizes a marching procedure, i.e., starting with the boundary conditions at one side of the system and successively marching along the structure to the other side of the system. The satisfaction of the boundary conditions at all boundary points provides the basis for solution's

*Corresponding author. E-mail: cb_gan@zju.edu.cn or cb_gan@yahoo.com. 


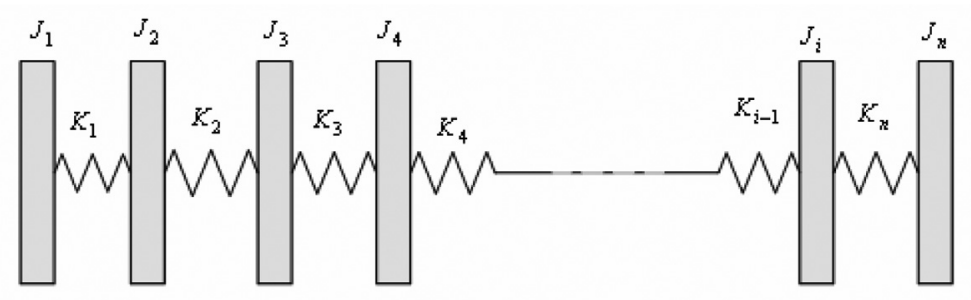

Fig. 1. Multi-segment lumped mass model.

location, and the state of the rotor system at a specific point is transferred between successive points through transfer matrices. This method is particularly suitable for "chainlinked" structures such as rotor systems, and primarily, it does not require the storage and manipulation of large system arrays [2].

The TMM was firstly proposed by Prohl [3]. Till now, there have been many contributions on the torsional vibration characteristics [4-9]. Pestel and Leckie [10] provided a thorough reference for applying the TMM to determine the natural frequencies and mode shapes for torsional systems. Rao [11] employed the TMM to analyze the free vibration, transient response, critical speed, and instability of the torsional rotor system. In Ref. [12], Lee et al. improved the TMM of the continuous-systems to fit the synchronous elliptical orbits of the linear rotorbearing systems by doubling the number of state variables. Their study also considered effects of the rotary inertia, gyroscopic and transverse shear. Furthermore, the TMM for continuous systems was extended to the unbalancing shaft [13] and the asymmetric rotor-bearing systems [14]. Aleyaasin et al. [15] applied the TMM to perform the flexural vibration analysis of a rotor mounted on fluid film bearings. Chen [16] investigated the torsional vibrations of a cylinder with varying cross-section and adhesive masses. Koser and Pasin [17] discussed the torsional vibrations of the drive shafts and mechanisms by means of analytical approach. In addition, the TMM and the Lagrangian dynamical method were developed to investigate the torsional and lateral vibrations of shafting systems in $[18,19]$.

Customarily, there are two kinds of direct time-integration method to obtain the transient response of a dynamical system. The first one is the explicit method such as the Runge-Kutta method, and the second one is the implicit method such as the Newmark method. Among the implicit methods, the Newmark method is based on the average acceleration and used most widely, which can guarantee numerical stability and have the second-order accuracy [20]. In Ref. [21], the Newmark- $\beta$ method combining with the Newton-Raphson approach was used to solve the nonlinear differential equations iteratively.

In this work, a modified Riccati torsional transfer matrix method is proposed. Combining with the Newmark- $\beta$ method, it is applied to study the characteristic frequencies and responses of the torsional vibrations in the turbogenerator shaft during power network faults or disturbance. In Section 2, the shafting system is modeled as a chain consisting of an elastic spring with concentrated mass points, and the modified Riccati transfer matrix method is then employed to derive the equations of characteristic frequencies. In Section 3, the modified matrix method is further applied to solve the responses of the torsional vibration of shafts combining with the Newmark- $\beta$ method. In Section 4, an experimental shafting system is set up to validate our approach. In the final section, the results of this work are summarized and discussed.

\section{Modified riccati torsional transfer matrix}

\subsection{Shafting system model}

According to the characteristic of a shaft's structure, a shafting system can be discretized into $n$ torsional vibration units, and the center line connecting all the units with large inertia is the center of the system. From Fig. 1, the shaft is now equivalent to a multi-segment lumped mass model. It consists of a uniform, free-free torsional bar with the length $L$ and torsional rigidity $K_{i}$, carrying $n$ mass moment of inertia $J_{i}(i=1,2, \ldots n)$. The interest here lies in establishing a relationship between the $n$ eigenfrequencies of this torsional vibration system.

The equation of motion of the system shown in Fig. 1 can be written in the following classical form: 


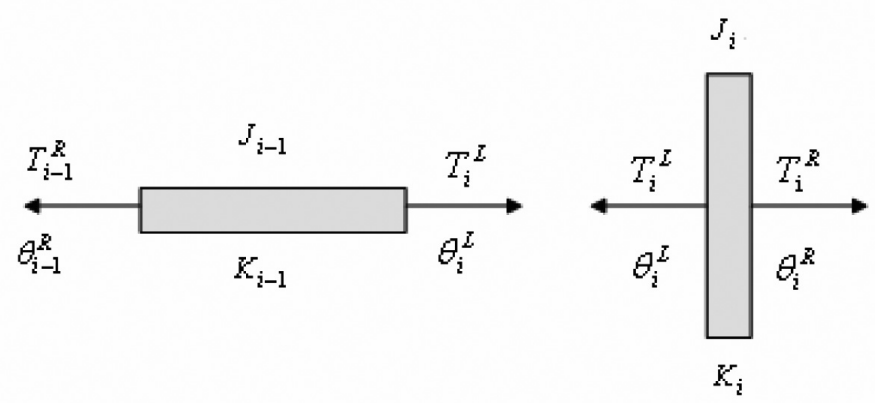

Fig. 2. Dynamical characteristic units.

$$
\mathbf{J} \ddot{\theta}(t)+\mathbf{K} \theta(t)=\mathbf{T}_{t}
$$

where $\mathbf{T}_{t}$ represents the external torque acting on the shaft, $\theta$ and $\ddot{\theta}$ represent the rotational angular displacement and angular acceleration respectively, $\mathbf{J}$ and $\mathbf{K}$ represent the angular moment of inertia and the rigidity matrix, respectively. When the external torque $\mathbf{T}_{t}=0$, the natural frequencies and the mode shapes can be readily obtained from the following equation:

$$
\mathbf{J} \ddot{\theta}(t)+\mathbf{K} \theta(t)=0
$$

\subsection{Riccati torsional transfer matrix}

In Fig. 2, the system is further divided into a series of dynamical characteristic units. The state vector of the endpoint represents the torsional vibration state of the system, while the transfer matrix represents the relationship between the state vectors of the adjacent elements.

The equation of motion of the $i$-th disk shown in Fig. 2 can be written as

$$
\mathrm{J}_{i} \ddot{\theta}_{i}=T_{i}^{R}-T_{i}^{L}
$$

where the state vector $\theta_{i}$ is the $i$-th torsional angular displacement, $T_{i}$ is the $i$-th internal torsional torque, $T_{i}^{R}$ represents the $i$-th internal torsional torque in the right side, and $T_{i}^{L}$ represents the $i$-th internal torsional torque in the left side. The relationship between the variables in the left and right sides has the following form

$$
\left[\begin{array}{l}
\theta \\
T
\end{array}\right]_{i}^{R}=\left[\begin{array}{cc}
1 & 0 \\
-\omega^{2} J_{i} & 1
\end{array}\right]\left[\begin{array}{l}
\theta \\
T
\end{array}\right]_{i}^{L}
$$

where

$$
T_{i}^{L}=T_{i-1}^{R}
$$

and

$$
\theta_{i}^{L}=\theta_{i-1}^{R}+\frac{T_{i-1}}{K_{i-1}}
$$

Substituting Eqs (5) and (6) into Eq. (4) yields

$$
\left[\begin{array}{l}
\theta \\
T
\end{array}\right]_{i}^{R}=\left[\begin{array}{ll}
1 & \frac{1}{K_{\mathrm{i}}-1} \\
-p^{2} J_{i} & 1-\frac{p^{2} J_{i}}{K_{i-1}}
\end{array}\right]\left[\begin{array}{l}
\theta \\
T
\end{array}\right]_{i-1}^{R}
$$

where $p$ is the angular frequency. When we introduce the Riccati transfer matrix $[S]_{i}:\{f\}_{i}^{R}=[S]_{i}\{e\}_{i}^{R}$, $\{Z\}_{i}=\left\{\begin{array}{l}\mathrm{e} \\ f\end{array}\right\}_{i}^{R},\{e\}_{i}=\theta_{i},\{f\}_{i}=T_{i}$, Eq. (7) is then replaced by

$$
\left[\begin{array}{l}
e \\
f
\end{array}\right]_{i+1}^{R}=\left[\begin{array}{ll}
U_{11} & U_{12} \\
U_{21} & U_{22}
\end{array}\right]_{i}\left[\begin{array}{l}
e \\
f
\end{array}\right]_{i}^{R}
$$




$$
[S]_{i+1}=\frac{\{f\}_{i+1}^{R}}{\{e\}_{i+1}^{R}}=\frac{U_{21 i}+U_{22 i}[S]_{i}}{U_{11 i}+U_{12 i}[S]_{i}}
$$

Equation (9) is the recurrence relation equation which can be used to calculate the natural frequency of the torsional vibration for a shafting system. With a certain calculating step, there must be a zero point between the residual values for the trial frequencies if the signs of two residual values are different, that is to say, there exits a correct root of the frequency equation between the two trial frequencies, which could be obtained from differential calculation. When the natural frequency is confirmed, its corresponding mode shape can also be deduced from Eq. (7).

\subsection{Improved riccati torsional transfer matrix}

In the use of Riccati method, the frequency equation usually leads to infinite singular points owing to the division operation, which influence the effectual calculation of the natural frequency. For the sake of eliminating the infinite singular points, Eq. (9) is improved by

$$
R=[S]_{i+1} \cdot \prod_{i=1}^{N} \frac{U_{11 i}+U_{12 i}[S]_{i}}{a b s\left(U_{11 i}+U_{12 i}[S]_{i}\right)}=0
$$

where $\mathrm{N}$ is the total number of characteristic units (see Figs 1 and 2), the symbol " $\Pi$ " denotes the multiply operator, and " $a b s(x)$ " means the absolute value of " $x$ ". The improved characteristic Eq. (10) can not only retain the advantages of the classical transfer matrix, but also avoid to lose the intrinsic frequency or add any nonexistent frequency due to the interference of infinite singular points.

\section{Incremental transfer matrix method}

\subsection{Incremental equation}

Rotor system is a highly complex nonlinear vibration system. For such a system, classical vibration theories and perturbation methods are no longer applicable. Currently, there are two categories of methods to calculate the transient vibration response of a rotor, i.e., the TMM and the mode synthesis method, both are supplemented by numerical integration. However, they can not be directly applied to study the torsional vibration of a rotor system.

In this subsection, we combine the Newmark- $\beta$ method with the TMM to establish the incremental equations of the rotor system. The Newmark- $\beta$ method is an integration method, and can be used to solve nonlinear equations effectively. The iteration algorithm from this method is usually expressed by the acceleration and velocity as

$$
\begin{aligned}
& \ddot{q}_{t+\Delta t}=\frac{1}{\beta \Delta t^{2}}\left(q_{t+\Delta t}-q_{t}\right)-\frac{1}{\beta \Delta t} \dot{q}_{t}-\left(\frac{1}{2 \beta}-1\right) \ddot{q}_{t} \\
& \dot{q}_{t+\Delta t}=\dot{q}_{t}+\frac{\gamma}{\beta \Delta t}\left(q_{t+\Delta t}-q_{t}\right)-\frac{\gamma}{\beta} \dot{q}_{t}-\left(\frac{\gamma}{2 \beta}-1\right) \ddot{q}_{t} \Delta t
\end{aligned}
$$

where $\gamma$ and $\beta$ are the parameters of the Newmark- $\beta$ function, $q$ is the generalized coordinate. From Eqs (11)-(12), the incremental equations are written as

$$
\begin{aligned}
& \Delta \ddot{q}_{t+\Delta t}=\ddot{q}_{t+\Delta t}-\ddot{q}_{t}=\frac{1}{\beta \Delta t^{2}}\left(\Delta q_{t+\Delta t}\right)-\frac{1}{\beta \Delta t} \dot{q}_{t}-\left(\frac{1}{2 \beta}\right) \ddot{q}_{t} \\
& \Delta \dot{q}_{t+\Delta t}=\dot{q}_{t+\Delta t}-\dot{q}_{t}=\frac{\gamma}{\beta \Delta t}\left(\Delta q_{t+\Delta t}\right)-\frac{\gamma}{\beta} \dot{q}_{t}-\left(\frac{\gamma}{2 \beta}-1\right) \ddot{q}_{t} \Delta t
\end{aligned}
$$

Now, Eqs (13)-(14) describe the incremental changes of the velocity and acceleration during the given time interval $\Delta t$, and can be applied to solve nonlinear equations. 


\subsection{Incremental transfer equations of torsional vibration}

From Fig. 1, the typical shafting system can be modeled as multi-disks. Each disk is acted by the inertia torque, the resistance torque, the torques from the left and right cross-sections, and the external torque. According to the moment equilibrium condition, the incremental equation is expressed as

$$
\Delta T_{i}^{R}(t+\Delta t)=\Delta T_{i}^{L}(t+\Delta t)+J_{i} \Delta \ddot{\theta}(t+\Delta t)+C_{i} \Delta \dot{\theta}(t+\Delta t)-\Delta T_{t i}(t+\Delta t)
$$

where $\Delta \theta_{i}^{R}=\Delta \theta_{i}^{L}, C_{i}$ is the resistance coefficient, and $\Delta T_{t i}$ is the inertia torque.

Substituting Eqs (13)-(14) into (15) yields

$$
\Delta T_{i}^{R}=\Delta T_{i}^{L}+A_{i} \cdot \Delta \theta_{i}^{L}+B_{i}
$$

where

$$
\begin{aligned}
A_{i}= & \frac{J_{i}}{\beta \cdot \Delta t^{2}}+\frac{C_{i} \gamma}{\beta \cdot \Delta t}, B_{i}=-J_{i}\left(\frac{1}{\beta \cdot \Delta t} \cdot \ddot{\theta}_{i}+\frac{1}{2 \beta} \cdot \ddot{\theta}_{i}\right)-C_{i}\left[\frac{\gamma}{\beta} \cdot \dot{\theta}_{i}+\left(\frac{\gamma}{2 \beta}-1\right) \cdot \ddot{\theta}_{i} \Delta t\right] \\
& -\Delta T_{t i}(t+\Delta t)
\end{aligned}
$$

For massless elastic shaft section, the incremental relation is as follows

$$
\left\{\begin{array}{l}
\Delta T_{i}^{R}=\Delta T_{i+1}^{L} \\
\Delta \theta_{i+1}^{L}=\Delta \theta_{i}^{R}+\frac{\Delta T_{i}^{R}}{K_{i}}
\end{array}\right.
$$

In respect to Eq. (8), for the i-th disk, if we denote $f_{i} \equiv \Delta T_{i}$ and $e_{i} \equiv \Delta \theta_{i}$, then the transfer matrix can be written as

$$
\left\{\begin{array}{l}
f \\
e
\end{array}\right\}_{i+1}^{L}=\left[\begin{array}{ll}
U_{11} & U_{12} \\
U_{21} & U_{22}
\end{array}\right]\left\{\begin{array}{l}
f \\
e
\end{array}\right\}_{i}^{L}+\left\{\begin{array}{l}
F_{f} \\
F_{e}
\end{array}\right\}_{i}
$$

where $U_{11}=1, U_{12}=A_{i}, U_{21}=1 / K_{i}, U_{22}=1+A_{i} / K_{i}, F_{f i}=B_{i}, F_{e i}=B_{i} / K_{i}$.

Substituting the Riccati transfer relation $f_{i}^{L}=S_{i} e_{i}^{L}+P_{i}$ into Eq. (19), we have

$$
\left\{\begin{array}{l}
S_{i+1}=\left[U_{11 i} S_{i}+U_{12 i}\right] \cdot\left[U_{21 i} S_{i}+U_{22 i}\right]^{-1} \\
P_{i+1}=\left[U_{11 i} P_{i}+F_{f i}\right]-S_{i+1}\left[U_{21 i} P_{i}+F_{e i}\right] \\
e_{i}^{L}=\left[U_{21 i} S_{i}+U_{22 i}\right]^{-1} e_{i+1}^{L}-\left[U_{21 i} S_{i}+U_{22 i}\right]^{-1}\left[U_{21 i} P_{i}+F_{e i}\right]
\end{array}\right.
$$

Then, the transfer matrices of $S_{i}, P_{i}$ and $e_{i}^{L}$ can be derived from Eq. (20).

\section{An illustrating example}

\subsection{Natural frequencies of torsional vibration}

Figure 3 shows the shafting system of a turbo-generator, where the length unit is $\mathrm{mm}$. This physical shafting system can adopt the rotor structure to simulate the high-pressure cylinder rotor, the low pressure cylinder rotor, the generator rotor, and the main exciter rotor. This simulated shaft is a multiple-rotor spring-mass system as shown in Fig. 1. Here, the total number of characteristic units is $n=163$.

The torsional natural frequencies of the shafting system are calculated through Eq. (9), where the shafting system is assumed to be free-free for the torsional vibration, and the boundary conditions on the start section are $\{e\}_{1}=\theta_{1} \neq 0$ and $\{f\}_{1}=T_{1}=0$, while those on end section are $\{e\}_{n}=\theta_{n} \neq 0$ and $\{f\}_{n}=T_{n}=0$. So, we have $[S]_{1}=\frac{\{f\}_{1}}{\{e\}_{1}}=0$ and $[S]_{n}=\frac{\{f\}_{n}}{\{e\}_{n}}=0$.

The first five natural frequencies for the torsional vibration of the shaft are shown in Table 1, where the frequencies by Eq. (9) are consistent with the design values. So, the improved Riccati transfer matrix method is very effective. In Fig. 4, we present the mode shapes corresponding to the first three natural frequencies. 
Table 1

The natural frequencies of the torsional vibration for the shaft

\begin{tabular}{lccccc}
\hline Order & 1 & 2 & 3 & 4 & 5 \\
\hline Calculation $(\mathrm{Hz})$ & 7.05 & 26.08 & 41.23 & 47.31 & 72.08 \\
Design Value $(\mathrm{Hz})$ & 7.35 & 25.75 & 40.55 & 46.75 & 71.75 \\
\hline
\end{tabular}

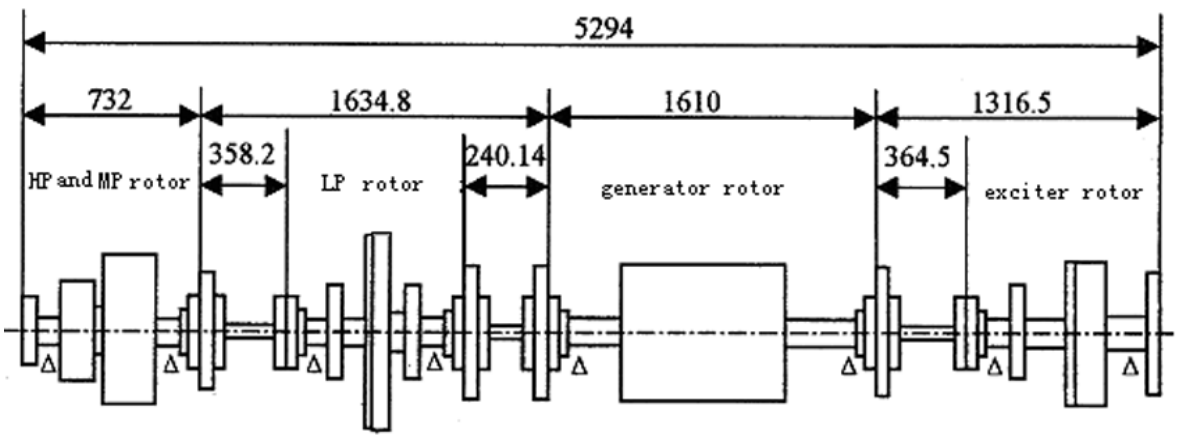

Fig. 3. The shaft structure of a turbo-generator, length unit: $\mathrm{mm}$.

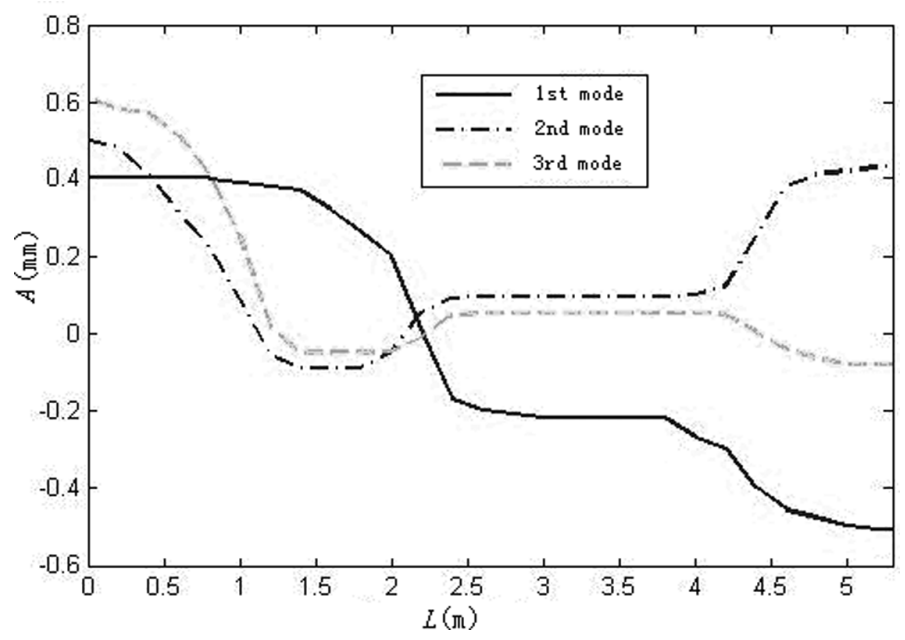

Fig. 4. The first three natural torsional modes of the shaft.

\subsection{Responses of torsional vibration under electrical disturbances}

To calculate the responses of the torsional vibration of the shaft, we firstly establish the physical parameters $J_{i}, C_{i}, K_{i}$, and the external torque increment $\Delta T_{t i}$ as shown in Fig. 1. For the sake of abbreviation, these parameters' values are not presented here. For the free-free shaft, the boundary conditions for the left and the right sections are $f_{1}^{L}=0, e_{1}^{L} \neq 0, S_{1}=0, P_{1}=0$ and $f_{n}^{R}=f_{n}^{L}+A_{n} e_{n}^{L}+B_{n}=0$ respectively, where $f_{n}^{L}=S_{n} e_{n}^{L}+P_{n}$, $e_{n}^{L}=-\left(P_{n}+B_{n}\right) /\left(S_{n}+A_{n}\right)$. Substituting the boundary conditions into Eq. (19), we can obtain the angular displacement increment $e_{i}^{L}(i=n, n-1, \cdots, 1)$ of each node and the torque increment $f_{i}^{L}$ of each section from right to left at the instant $t+\Delta t$ by the recursive formula.

In general, the short-circuit fault can bring a strong impact on the short-circuit current and the torque in the vicinity of a power system, especially in the generator outlet. The electromagnetic torque of two-phase and three-phase short-circuit can be described as in Ref. [21]. Equation (21) is the electromagnetic torque for three-phase shortcircuit, its characteristic in time-domain is displayed in Fig. 5(a). While Eq. (22) is the electromagnetic torque for two-phase short-circuit, its characteristic in time-domain is displayed in Fig. 5(b). 


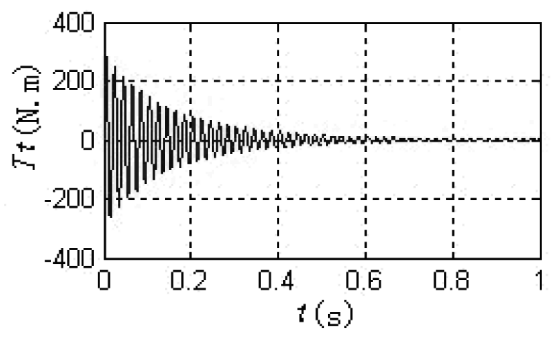

(a) Three-phase short-circuit

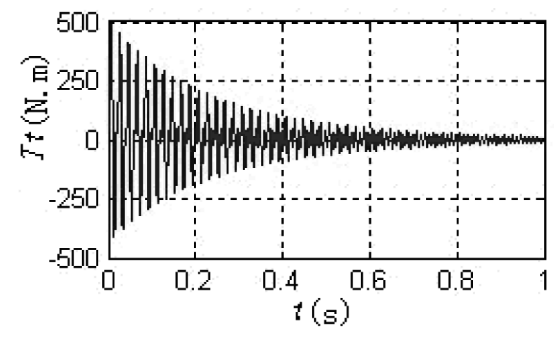

(b) Two-phase short-circuit

Fig. 5. Characteristics of the electromagnetic torques under the short-circuit faults.

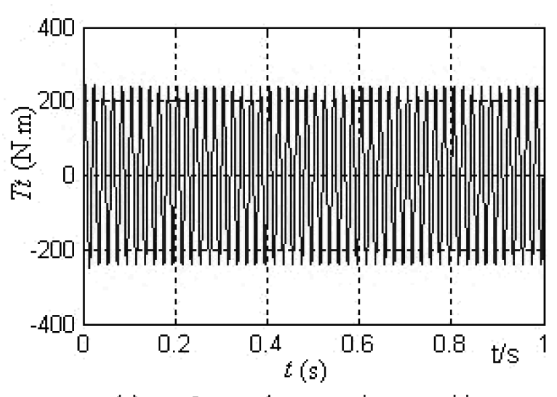

(a) $120^{\circ}$ asynchronous juxtaposition

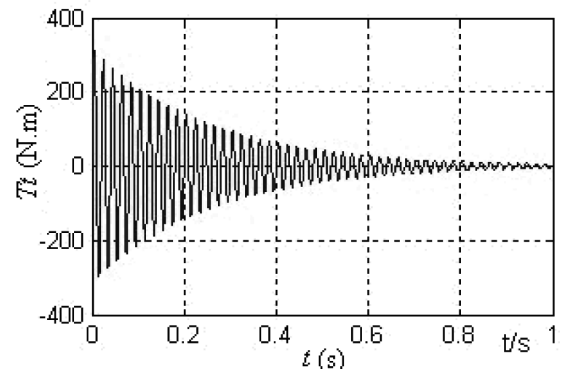

(b) $180^{\circ}$ asynchronous juxtaposition

Fig. 6. Characteristics of the electromagnetic torques under the asynchronous juxtapositions.

$$
T_{t}(x, t)=M_{0}\left(A M e^{-\alpha t} \sin \omega t+B M^{2}\right)
$$

where $A=6.259, B=0.0877, \alpha=3.9809, \omega=2 \pi n_{1} / 60, M_{0}$ is the rated torque of the generator under the normal operation, $M=0.07954+0.563 e^{-1.1443 t}+0.3569 e^{-9.1575 t}$, and $n_{1}$ is the speed of the rotor, see [21]. So,

$$
T_{t}(x, t)=M_{0}\left(A e^{-\alpha t} \sin \omega t-B e^{-\beta t} \sin 2 \omega t+C e^{-\gamma t}\right)
$$

where $A=7.591, B=3.795, C=0.742, \alpha=5.305, \beta=2.65, \gamma=4.428$, and the other parameters are the same as those in Eq. (21).

The asynchronous juxtaposition with a strong impact on the current and the torque is one of the typical electrical interferences during the power operation. The electromagnetic torques of $120^{\circ}$ and $180^{\circ}$ asynchronous juxtapositions can be described as Eqs (23)-(24), where Eq. (23) is the electromagnetic torque for $120^{\circ}$ asynchronous juxtaposition, its characteristic in time-domain is displayed in Fig. 6(a), while Eq. (24) is the electromagnetic torque for $180^{\circ}$ asynchronous juxtaposition, its characteristic is displayed in Fig. 6(b).

$$
T_{t}(x, t)=M_{0}\left(M_{1}(t) \cdot M_{2}(t)+M_{3}(t)\right)
$$

where $M_{1}(t)=2.796 \times\left(0.2226 e^{-7.5175 t}+0.634 e^{-0.876 t}+0.7774\right), M_{2}(t)=e^{-9.1575 t} \cos \omega t, M_{3}(t)=$ $5.004 \sin \omega t-2.796 e^{-3.981 t} \cos \omega t$. So,

$$
T_{t}(x, t)=M_{0} A e^{-\alpha t} \sin \omega t
$$

where $A=6.6172, \alpha=3.9809$, and the other parameters are the same as those in Eq. (21).

In a variety of short-circuit faults, the two-phase or three-phase short-circuit of the generator outlet is more serious for the shaft. The electromagnetic torque of the two-phase short-circuit shown in Fig. 6(b) is larger than that of the three-phase short-circuit shown in Fig. 6(a). Figures 7 and 8 are the dynamical responses and their corresponding frequencies of the torsional vibration under the network short circuit faults. The response trail of the torsional vibration under the two-phase short-circuit in Fig. 8(a) is similar to that under the three-phase short-circuit in Fig. 7(a), and the fast Fourier transform (FFT) plots shown in Figs 7(b) and 8(b) indicate that both the responses have the same frequencies, i.e., $7.0 \mathrm{~Hz}, 26.2 \mathrm{~Hz}, 41.4 \mathrm{~Hz}, 47.6 \mathrm{~Hz}, 50 \mathrm{~Hz}$ and $72.1 \mathrm{~Hz}$, among which the frequency $50 \mathrm{~Hz}$ 


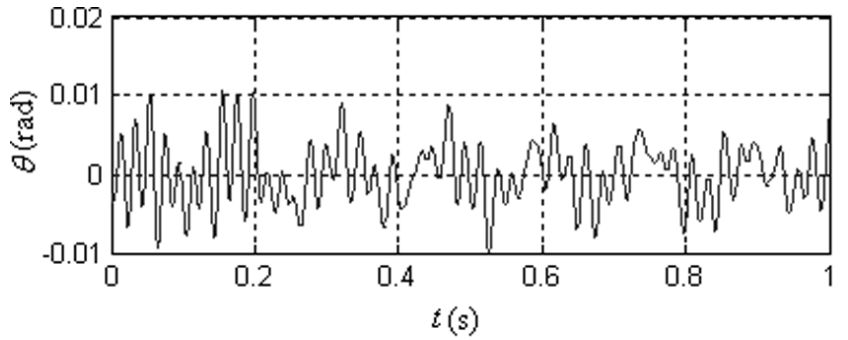

(a) Time response of torsional vibration

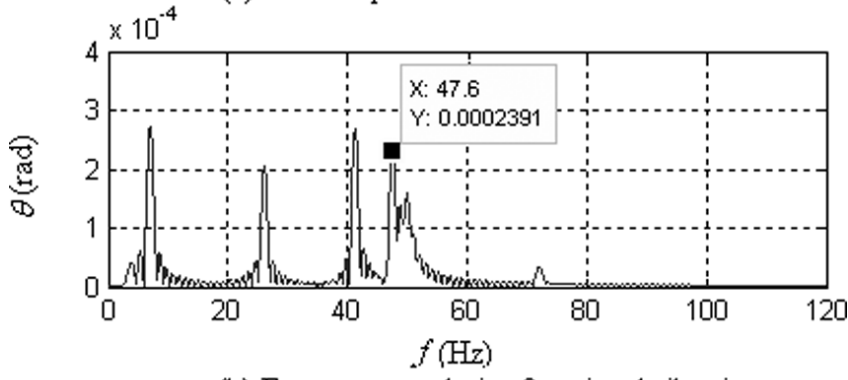

(b) Frequency analysis of torsional vibration

Fig. 7. Response and frequency analysis of the torsional vibration under the three-phase short-circuit.

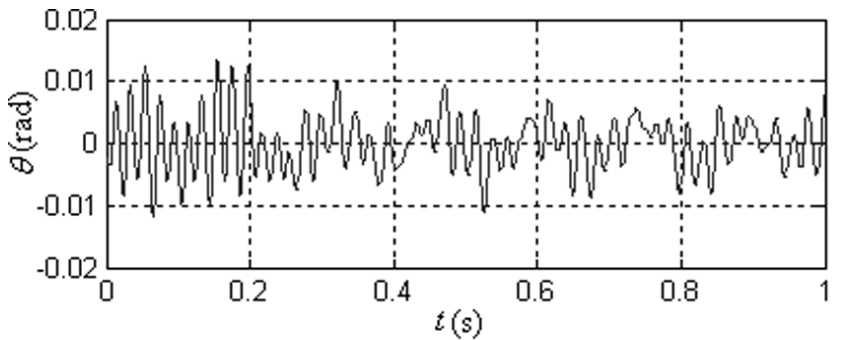

(a) Time response of torsional vibration

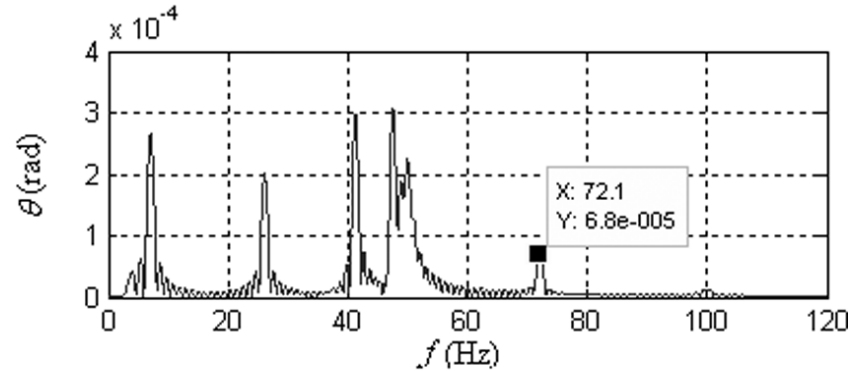

(b) Frequency analysis of torsional vibration

Fig. 8. Response and frequency analysis of the torsional vibration under the two-phase short-circuit.

is the rotating frequency of rotor. However, the maximal torsional angular displacement excited by the two-phase short-circuit is larger than that under the three-phase short-circuit. In addition, the $2 \mathrm{X}$ rotating frequency component $(100 \mathrm{~Hz})$ appears in the spectrum diagram under the two-phase short-circuit fault Fig. 8(b), but its amplitude is very small. That is to say, the torsional vibration owing to the two-phase short-circuit fault is stronger than that owing to the three-phase short-circuit fault.

From Figs 6(c) and 6(d), the electromagnetic torque under $120^{\circ}$ asynchronous juxtaposition is different from that under $180^{\circ}$ asynchronous juxtaposition. In Figs 9-10, we present the dynamical responses and their corresponding frequencies of the torsional vibration of the shaft under the asynchronous juxtaposition. It is shown that, the response 


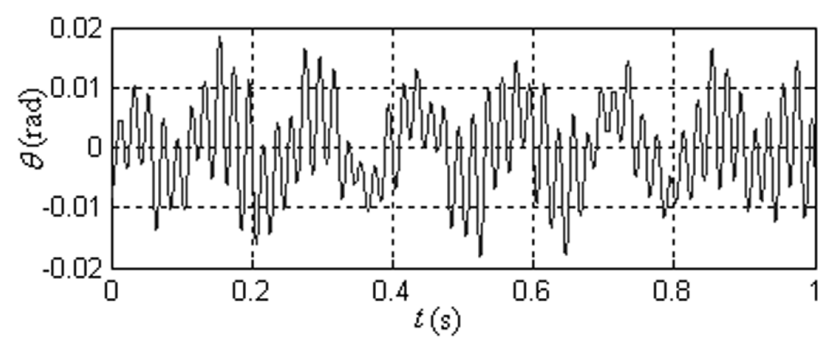

(a) Time response of torsional vibration

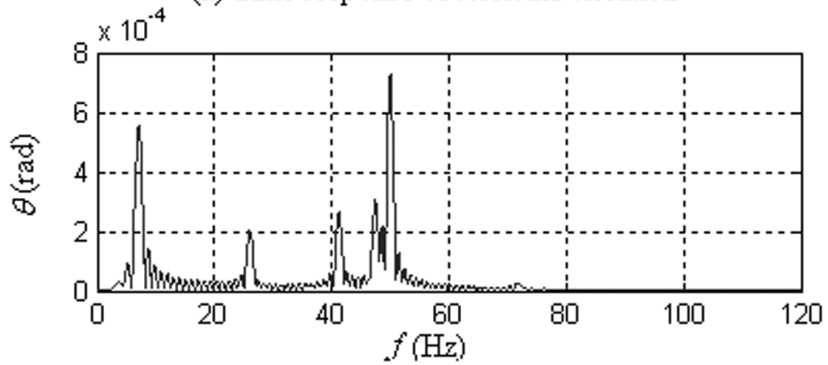

(b) Frequency analysis of torsional vibration

Fig. 9. Response and frequency analysis of the torsional vibration under $120^{\circ}$ asynchronous juxtaposition.

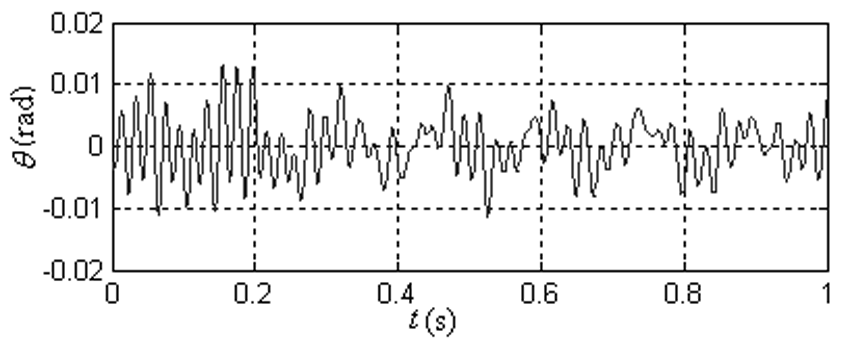

(a) Time response of torsional vibration

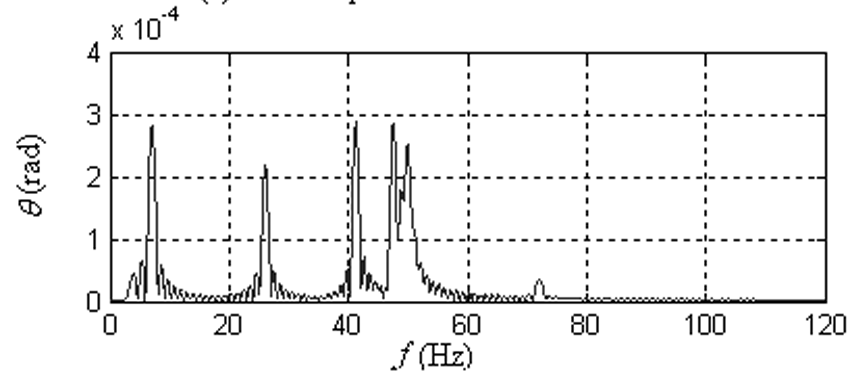

(b) Frequency analysis of torsional vibration

Fig. 10. Response and frequency analysis of the torsional vibration under $180^{\circ}$ asynchronous juxtaposition.

trail of the torsional vibration under $120^{\circ}$ asynchronous juxtaposition in Fig. 9(a) is different from that under $180^{\circ}$ asynchronous juxtaposition in Fig. 10(a), and the maximal torsional angular displacement under $120^{\circ}$ asynchronous juxtaposition is larger. Nevertheless, both can provoke the first five natural frequencies $(7.0 \mathrm{~Hz}, 26.2 \mathrm{~Hz}, 41.4 \mathrm{~Hz}$, $47.6 \mathrm{~Hz}, 72.1 \mathrm{~Hz}$ ) of the shaft, and these natural frequencies are approximately equal to the calculated values shown in Table 1. In addition, the rotating frequency of the rotor $(50 \mathrm{~Hz})$ also appears in Figs 9(b) and 10(b). From Figs 9-10, the asynchronous juxtaposition has a stronger impact on the current and the torque. When closing about $120^{\circ}$ angle, the electromagnetic torque can approach the maximum, and the torsional vibration under $120^{\circ}$ asynchronous juxtaposition is serious (see Fig. 9). In Fig. 11, the root mean square (RMS) values of the torsional 


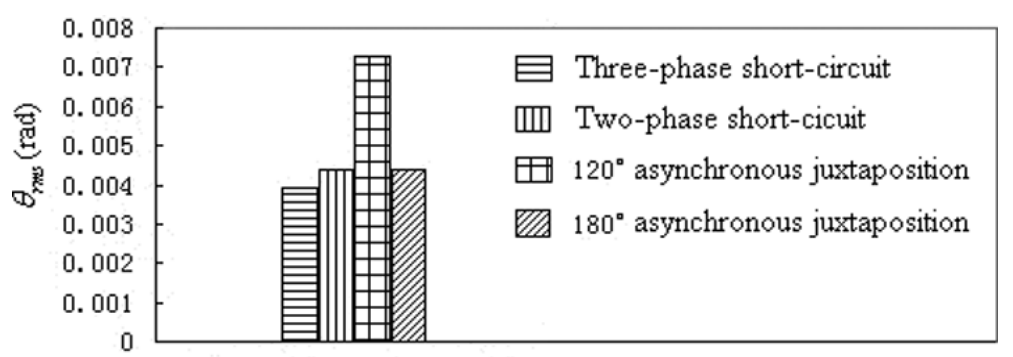

Fig. 11. Comparison between the RMS values of the torsional vibration during the network faults.

vibration responses for four network disturbances are compared with each other, from which the torsional vibration under $120^{\circ}$ asynchronous juxtaposition is the most serious.

\section{Conclusions and discussions}

In this work, a highly complex shafting system is analyzed by a modified Riccati torsional transfer matrix combining with the Newmark- $\beta$ method. The system is modeled as a chain consisting of an elastic spring with concentrated mass points, and the multi-segments lumped mass model is established for this shafting system. By the modified Riccati torsional transfer matrix method, an accumulated calculation is effectively eliminated to obtain the natural frequencies and the responses of the torsional vibration for the shaft. Furthermore, we combine the Newmark- $\beta$ method with the modified Riccati torsional transfer matrix to establish the incremental transfer matrix, which is used to solve the nonlinear equations for the torsional vibration of the shaft.

Based on the multi-segments lumped mass model and the incremental transfer matrix method, we have derived the calculation matrix for the response of the torsional vibration. Taking the torsional vibration simulator for a turbinegenerator as the object, comparisons between the simulation results and the design values show that the incremental transfer matrix method can be effectively applied to study the torsional vibration characteristics. Different transient responses of the shaft's torsional vibrations were simulated under some typical power network disturbances, such as the three-phase short circuit, the two-phase short circuit, and the asynchronous juxtaposition. All these disturbances can excite the natural frequencies of the former five modes, but the torsional vibration is the most serious under $120^{\circ}$ asynchronous juxtaposition. We believe that the present study is instructive for shafting system designers.

\section{Acknowledgements}

The authors acknowledge the supports from the National Natural Science Foundation of China under Grant Nos. 11072078 and 11072214, the State 863 Plan of China under Grant No. 2008AA04Z410, and the open project of the State Key Laboratory of Mechanical System and Vibration of China under Grant No. MSV-2010-14.

\section{References}

[1] A.D. Dimarogonas, Vibration of cracked structures: a state of the art review, Engineering Fracture Mechanics 55(5) (1996), 831-857.

[2] F.F. Ehrich, Handbook of Rotordynamics, McGraw-Hill, New York, 1992.

[3] M.A. Prohl, A general method for calculating critical speeds of fiexible rotors, Journal of Applied Mechanics 12 (1945), 142-148.

[4] P.N. Bansal and R.G. Kirk, Stability and damped critical speeds of rotor-bearing systems, Journal of Engineering for Industry 97 (1975), $1325-1332$.

[5] E.S. Zorzi and H.D. Nelson, Finite element simulation of rotor-bearing systems with internal damping, Journal of Engineering for Power 99(A1) (1977), 71-76.

[6] W.D. Pilkey and P.Y. Chang, Modern Formulas for Statics and Dynamics: A Stress-and-Strain Approach, McGraw-Hill, New York, 1978.

[7] S. Sankar, On the torsional vibration of branched systems using extended transfer matrix method, Journal of Mechanical Design 101 (1979), 546-553. 
[8] J.W. Lund, Sensitivity of the critical speeds of a rotor to changes in the design, Journal of Mechanical Design 102 (1980), 115-121.

[9] K.B. Yim, S.T. Noah and J.M. Vance, Effect of tangential torque on the dynamics of fiexible rotors, Journal of Applied Mechanics $\mathbf{5 3}$ (1986), 711-718.

[10] E.C. Pestel and F.A. Leckie, Matrix Methods in Elastomechanics, McGraw-Hill, New York, 1963.

[11] J.S. Rao, Rotor Dynamics, Wiley Eastern, New Delhi, 1991.

[12] A.C. Lee, Y. Kang and S.L. Liu, A modified transfer matrix method for linear rotor-bearing systems, Journal of Applied Mechanics 58 (1991), 776-783.

[13] A.C. Lee, Y.P. Shih and Y. Kang, The analysis of linear rotor-bearing systems: a general transfer matrix method, Journal of Vibration and Acoustics 115 (1993), 490-496.

[14] S.C. Hsieh, J.H. Chen and A.C. Lee, A modified transfer matrix method for the coupled lateral and torsional vibrations of asymmetric rotor-bearing systems, Journal of Sound and Vibration 312 (2008), 563-571.

[15] M. Aleyaasin, M. Ebrahimi and R. Whalley, Multivariable hybrid models for rotor-bearing systems, Journal of Sound and Vibration 233 (2000), 835-856.

[16] Y.Z. Chen, Torsional free vibration of a cylinder with varying cross-section and adhesive masses, Journal of Sound and Vibration 241(3) (2001), 503-512.

[17] K. Koser and F. Pasin, Torsional vibrations of the drive shafts of mechanisms, Journal of Sound and Vibration 199 (1997), $559-565$.

[18] J.S. Wu and I.H. Yang, Computer method for torsion-and-fiexural-coupled forced vibration of shafting system with damping, Journal of Sound and Vibration 180(3) (1995), 417-435.

[19] B.O. Al-Bedoor, Transient torsional and lateral vibrations of unbalanced rotors with rotor-to-stator rubbing, Journal of Sound and Vibration 229 (2000), 627-645.

[20] S.P. Harsha and P.K. Kankar, Stability analysis of a rotor bearing system due to surface waviness and number of balls, Journal of Mechanical Sciences 46 (2004), 1057-1081.

[21] K. Bathe and E. Wilson, Numerical methods in finite element analysis. Englewood Cliffs, NJ: Prentice-Hall, 1990.

[22] IEEE SSR Working Group. Second benchmark model for computer simulation of subsynchronous oscillations, IEEE Trans on PAS 104(5) (1985), 1057-1066. 

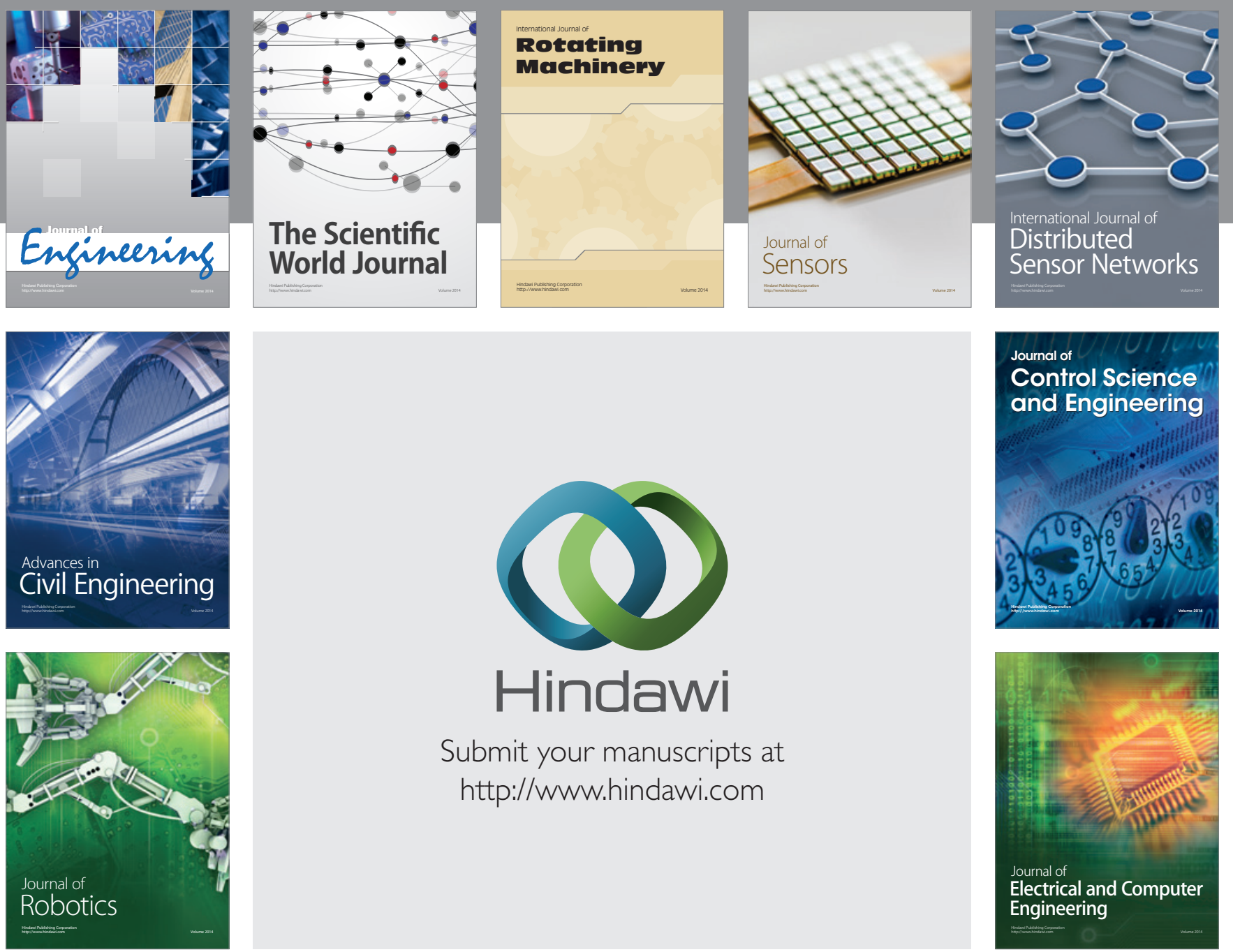

Submit your manuscripts at

http://www.hindawi.com
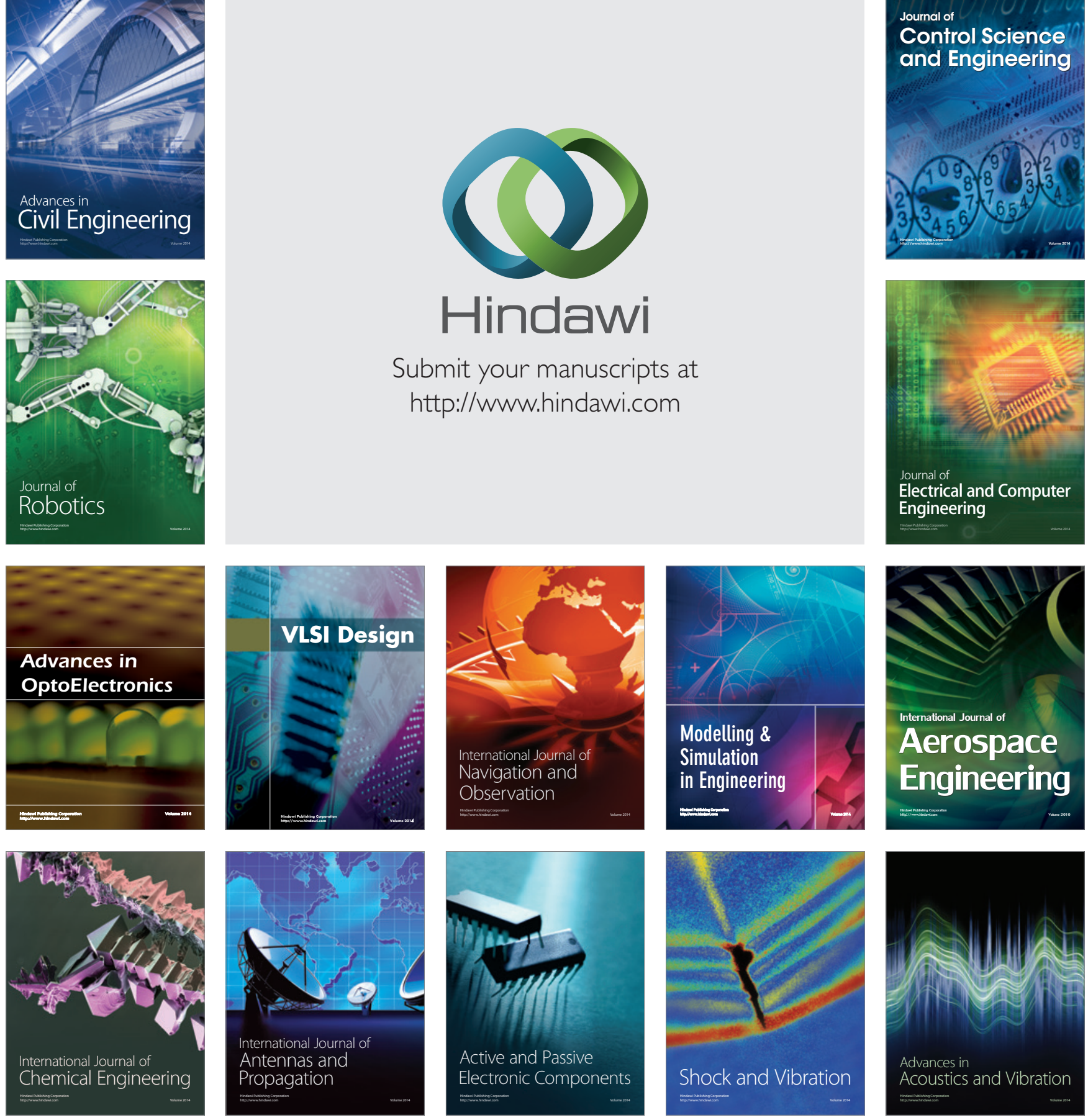